\title{
RECURRENT DENTAL INFECTION DUE TO USE OF SSRIS: A CASE REPORT
}

\author{
Soumitra Das \& Lokesh Sekharan \\ Melbourne Health, Hume Community Team, Melbourne, Australia
}

received: 9.2.2021;

revised: 6.3.2021;

$* * * * * *$

\section{INTRODUCTION}

Dental infection can cause significant pain leading poor sleep and quality of life. It can also induce secondary problems such as overuse of opioids and related complications, poor adherence to psychotropic and relapse. It is relatively expensive to afford dental procedure in a developed country causing significant financial burden. Dental infections among the mentally ill can be due to multiple causes, most common being poor hygiene, excessive use of sweet drinks, or dryness of mouth-related to anticholinergic medications (Kisely 2016). It is not very common to see repeated severe dental carries directly linked to the use of SSRI.

\section{CASE}

Here we present a unique case where 42 years old woman presented with long-standing anxiety and depression, subsequently developed severe dental infections only after starting the antidepressants. She was started on Paroxetine which caused dry mouth within a week. She then developed a dental infection within the same period which needed multiple courses of antibiotics over the next 2 months. She had to go for dental extraction twice in 12 weeks. As her dentist and general practitioner ruled out all the other causes like oral hygiene, pre-existing dental condition, excessive consumption of sweet drinks, and any other physical illness contributing to the infections, they considered the Paroxetine could be the contributing agent for her dental infection. She self-ceased her antidepressant and she was not having complaint of dental infection however her anxiety and depressive symptoms were gradually worsening in severity. Later her GP started Escitalopram to lessen the anxiety symptoms. This time she did not report dry mouth however she had a recurrence of dental infection. Once again she needed two courses of antibiotics however the infection severity was lesser than before. Later her antidepressant was changed to Desvelnafaxine as it had less potential to cause dryness of the mouth. Her depression and anxiety continued to improve without any recurrence of dental infection.

\section{DISCUSSION}

A person experiencing depression or anxiety, either on antidepressants or not, pose the risk of extensive tooth losses. It may occur due to multiple factors such as diminished interest in oral hygiene associated with anhedonia, craving for carbohydrates or sweetened foods because of alterations in the sense of taste or reduced serotonin level in the brain, depletion of saliva release, and the high lactobacillus counts (de Ameida et al. 2012). Antidepressants with an anticholinergic property like TCAs can cause significant dryness of the mouth (Scully 2003). A dry oral cavity can trigger candidosis. Also, lack of saliva can reduce the lubrication of teeth causing mucosal erosion in the dental socket. TCAs reduce in parotid flow, also decreases $\mathrm{Na}+$ and increases $\mathrm{K}+$ ions. SSRI does not have a significant difference from control regarding salivary flow (Hunter \& Wilson 1995). Also, exclusive intake of SSRIs does not alter the total salivary proteins, urea, and calcium concentrations, alpha-amylase activity, $\mathrm{pH}$, and salivary buffer capacity (SBC). However, Zimelidine, not so commonly used SSRI, can increase alpha-amylase activity or total protein contents, due to sympathomimetic effect, raising doubt about the selectivity of SSRIs. The effects of antidepressants on glandular secretion can follow a complex mechanism as these have effects on several levels, such as in the brain, in the proximal cholinergic synapses, and nerveeffector cleft. Also, the effects can depend on the period of treatment, differences in absorption and excretion rates, action, plasma concentration, drug dose, and active biologic metabolite formation (de Almeida et al. 2008). Also, the presence of xerostomia could due to secondary to the psychological process such as generalized anxiety or panic symptoms causing dryness of the mouth (Veerabhadrappa et al. 2016). The presence of xerostomia can trigger various clinical problems like mucosal dryness; difficulty in swallowing and speech; high susceptibility to oral infections, mainly candidosis and dental caries; gingivitis; and mucositis (Daly 2016). Also, a large number of people complain of dryness of mouth even with the newer antidepressants therefore, oral care is indispensable (de Almeida et al. 2008). 


\section{CONCLUSION}

Hence antidepressants can be directly linked to relatively rare side effects like a dental infection. It can be distressing to patients, which can influence the adherence to ongoing treatment. It is important to look into the relatively rare causative factors and choose an antidepressant accordingly.

\section{Acknowledgments: None.}

\section{Conflict of interest: None to declare.}

\section{Contribution of individual authors:}

Soumitra Das: reviewing the literature and writing the original draft.

Lokesh Sekharan: writing the original draft and editing.

\section{References}

1. de Almeida PDV, Grégio AMT, Brancher JA, Ignácio SA, Machado MÂN, de Lima AAS, et al.: Effects of antidepressants and benzodiazepines on stimulated salivary flow rate and biochemistry composition of the saliva. Oral Surgery, Oral Medicine, Oral Pathology, Oral Radiology and Endodontology 2008; 106:58-65

2. de Ameida PDV, Rodrigues Johann ACB, Azevedo Alanis LR de, de Lima AAS, Trindade Grgio AM: Antidepressants: Side Effects in the Mouth. In: Oral Health Care Pediatric, Research, Epidemiology and Clinical Practices. InTech, 2012

3. Daly C: Oral and dental effects of antidepressants. Vol. 39, Australian Prescriber. Australian Government Publishing Service 2016; 84

4. Hunter KD, Wilson WS: The effects of antidepressant drugs on salivary flow and content of sodium and potassium ions in human parotid saliva. Archives of Oral Biology 1995; 40:983-9

5. Kisely S: No mental health without oral health. Vol. 61, Canadian Journal of Psychiatry. SAGE Publications Inc 2016; 277-82

6. Scully C: Drug effects on salivary glands: Dry mouth. Vol. 9, Oral Diseases. John Wiley \& Sons, Ltd; 2003; 165-76

7. Veerabhadrappa SK, Chandrappa PR, Patil S, Roodmal SY, Kumarswamy A, Chappi MK: Evaluation of xerostomia in different psychological disorders: An observational study. Journal of Clinical and Diagnostic Research 2016; 10:ZC24

\section{Correspondence:}

Soumitra Das, MD, Psychiatry Registrar Melbourne Health, Hume Community Team Jacana, Broadmeadows 3047, Melbourne, Australia E-mail: soumitratdmc@gmail.com 